\title{
Posterior reversible encephalopathy syndrome - Case report
}

\author{
Marcin Kulczyński ${ }^{1, D-F} \oplus$, Gabriela Kuroska ${ }^{2, A-B, D \oplus}{ }^{\text {, Klaudia Sapko,C-D } \odot \text {, Michał Marciniec }}{ }^{1, C-D \oplus}$, \\ Esteban Muñoz-Niklitschek ${ }^{3, D-E \oplus}$, Przemysław Dyndor ${ }^{4, A-B, D \oplus}$, Katarzyna Dyndor ${ }^{5, A-D, F \oplus}$ \\ ${ }^{1}$ Chair and Department of Neurology, Medical University of Lublin, Poland \\ ${ }^{2}$ Chair and Department of Human Anatomy, Medical University of Lublin, Poland \\ ${ }^{3}$ Faculty of Medicine, University of Concepción, Chile \\ ${ }^{4}$ Chair and Department of Rehabilitation and Orthopaedics, Medical University of Lublin, Poland \\ ${ }^{5}$ Department of Radiography, Medical University of Lublin, Poland \\ A - Research concept and design, B - Collection and/or assembly of data, C - Data analysis and interpretation, \\ $D$ - Writing the article, E - Critical revision of the article, F - Final approval of article
}

\begin{abstract}
Kulczyński W, Kuroska G, Sapko K, Marciniec M, Muñoz-Niklitschek E, Dyndor P, Dyndor K. Posterior reversible encephalopathy syndrome Case report. J Pre-Clin Clin Res. 2020; 14(4): 123-125. doi: 10.26444/jpccr/128702
\end{abstract}

\begin{abstract}
Introduction. Posterior reversible encephalopathy syndrome (PRES) is a group of clinical disorders typically manifested by the presence of seizures, headache, impaired consciousness, nausea and focal neurological deficits. It is stated that PRES is a result of high blood pressure which leads to impaired self-regulation and hyperperfusion causing endothelial damage and vasogenic oedema or that excessive arteriolar vasoconstriction causes reduced blood flow, thus causing ischemia and cytotoxic oedema.

Case Report. A 21-year-old woman was admitted to obstetrics \& gynaecology department with symptoms of eclampsia. CT of the head revelaed a holohemispheric watershed pattern. MRI confirmed the presence of vasogenicoedema in the affected regions, typical for PRES. C-section was performed. Ten days after administering treatment, MRI showed complete withdrawal of the lesions. The diagnosis of PRES was based on clinical symptoms and MRI, with typical cortical and subcortical oedema without infarction.
\end{abstract}

\section{Key words:}

posterior reversible encephalopathy syndrome, PRES, vasogenic oedema

\section{INTRODUCTION}

Posterior reversible encephalopathy syndrome (PRES) is a clinical and radiological entity that was described for the first time by Hinchey in 1996. The global incidence of PRES is unknown. This syndrome has been reported among patients aged 4-90, although most cases occur in young and middleaged adults, although it may be more common in females. The typical clinical findings of PRES are epileptic seizures (even status epilepticus), headache, impaired consciousness, visual disturbances, nausea and focal neurological deficits $[1,2]$. Acute hypertension is not usually described among the main signs of PRES, but it is mentioned in most case studies. The diagnosis depends on clinical findings, with the supportive role of magnetic resonance imaging of the brain. Three different patterns have been described in MRI including: holohemispheric watershed pattern, superior frontal sulcus pattern and dominant parietal-occipital [3].

There are two main hypotheses for the pathophysiology of PRES. One involves impaired cerebral autoregulation responsible for an increase in cerebral blood flow (CBF), whereas the other involves endothelial dysfunction with accompanying cerebral hypoperfusion. The result of the cerebral blood perfusion abnormalities is the bloodbrain barrier dysfunction resulting in cerebral vasogenic oedema $[4,5]$. Several factors appear to play a role in the

Address for correspondence: Marcin Kulczyński, Chair and Department of Neurology, Medical University, Lublin, Poland

E-mail: mk.marcin.kulczynski@gmail.com

Received: 22.07.2020; accepted: 14.10.2020; first published: 29.10.2020 pathogenesis of PRES, with exposure to toxic agents being the most common condition associated with PRES. Arterial hypertension, infection, sepsis and septic state, eclampsia and pre-eclampsia, autoimmune diseases or renal failure are also associated with higher risk of PRES. Treatment strategy mainly involves supportive measures and removing the underlying causes. An appropriate treatment is expected to ensure a full recovery. Many cases resolve within 1-2 weeks from the initial diagnosis [6]; however, some cases take longer to recover, and may involve permanent neurologic deficits, such as vision disturbances and epileptic seizures. Fatal outcome has been reported in up to $15 \%$ of patients. PRES may recur in about 5-10\% of cases; this occurs more commonly in patients with arterial hypertension.

\section{CASE REPORT}

A 21-year-old primigravida in the 31st hbd (hebdomas graviditatis, week of pregnancy) was admitted to the Obstetrics \& Gynaecology Department, complained of stomachache, diarrhea and vomiting. Next morning, she presented generalized epileptic seizures. Urinalysis showed proteinuria with bacteria and leucocytes. Her complete blood count test showed a leukocyte count of $12.1 \mathrm{~K} / \mu \mathrm{l}$; serum CRP level was within the norms. Abdominal ultrasound showed a pregnancy in approx. $31^{\text {st }} \mathrm{hbd}$ by appearance. Patient's arterial blood pressure was 190/110 $\mathrm{mmHg}$.

An immediate cesarean section was performed, during which magnesium sulphate treatment was 


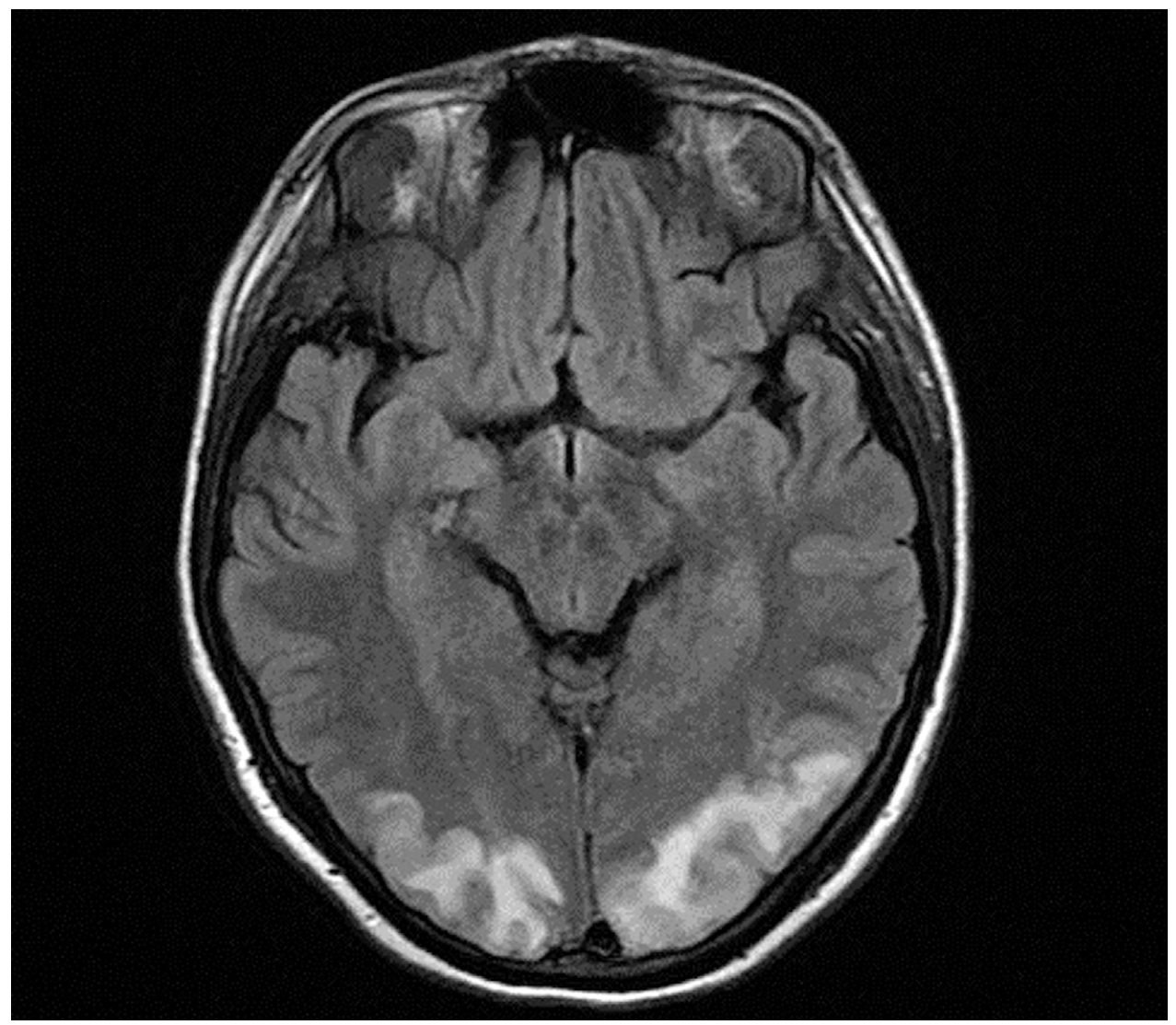

Figure 1. Fluid-attenuated inversion recovery (FLAIR) showing a developing vasogenic oedema

introduced. Another epileptic seizure was noticed. An infant weighing 2,100 grams with APGAR scores of eight in the first and fifth minute was delivered. Postpartum arterial blood pressure was $180 / 130 \mathrm{mmHg}$ with accompanying tachycardia. Patient received nitroglycerine. Additionally, she reported loss of vision, mainly in the right eye. Also, after cesarean section, position tremors of the upper limb were still observed. Brain CT showed a holohemispheric hypodense watershed pattern and dominant parieto-occipital. MRI confirmed the presence of vasogenic oedema in the affected regions (Fig. 1).

On the fourteenth day of hospitalization, vision impairment completely resolved, as well as the position tremor of the upper limb. MR scan of the brain showed complete resolution of the previously present lesions, and posterior reversible encephalopathy syndrome was diagnosed.

\section{DISCUSSION}

Diagnosing a patient with eclampsia who presents acute neurological symptoms and consciousness impairment remains remarkably difficult, with a significant role of MRI, greater than CT. Typical symptoms of PRES include consciousness impairment, epileptic seizures, vision disturbances, headaches, vomiting, nausea and focal neurological deficits, often being accompanied by high blood pressure. Consciousness impairment level can range from mild confusion to even coma. Seizures mostly appear as generalized tonic-clonic.

The presented patient showed almost all of the abovementioned symptoms, beginning with vomiting, increased blood pressure, seizures with loss of consciousness and vision disorders mainly in the right eye. Hinchey, who was the first to describe PRES in 1996, noted the relationship between eclampsia and posterior reversible encephalopathy syndrome. Three out of fifteen patients in his study showed this coexistence. Pre-eclampsia and eclampsia are common causes of PRES. Many authors, among the causes of reversible encephalopathy, also mention HIV infection, haemolytic uraemic syndrome, glomerulonephritis, hypercalcaemia, blood transfusions, and the use of corticosteroids, tacrolimus or cyclosporine A $[6,7]$.

Brain imaging shows that parieto-occipital and posterior part of the frontal lobes are predominantly involved. Head CT has a significantly lower sensitivity than the brain MRI which shows typical T2/FLAIR hyperintensities which are bilateral, and involvthe cortex and subcortical white matter. Affected areas also demonstrate restricted diffusion in DWI sequence, often with lesser extensiveness as compared to T2/ FLAIR images (Fig. 2).

Abnormalities may also be localized at the deep brain structures [3]. There are several theories explaining this pathology: increased permeability of the blood-brain barrier, failed autoregulation of cerebral circulation and epithelial injury, leading eventually to vasogenic cerebral oedema $[2,8,9]$. This type of oedema localized at parietooccipital region was demonstrated in the presented patient in the brain MRI. Vertebral/basiliar artery occlusion and venous sinus thrombosis should have been ruled out as possible causes of identified pathologies. Eclampsia and impaired autoregulation of cerebral circulation were the underlying cause of the patients' disorders which resolved 
after caesarean section and proper treatment of seizures and hypertension.

Early diagnosis and treatment allows a full recovery in a few weeks, most often without any permanent neurological deficits. Misdiagnosis or delayed treatment can lead to complications such as cerebral ischemia, stroke and even fatal outcome [10].

\section{CONCLUSIONS}

The diagnosis of PRES depends on clinical symptoms, with the supportive role of magnetic resonance imaging of the brain. Three different patterns have been described in MR scans, including holohemispheric watershed pattern, superior frontal sulcus pattern and dominant parietal-occipital. In the case of suspicion of PRES, a brain MRI should be performed, especially DWI and ADC sequences should be acquired. Although rare, PRES should be included in differential diagnosis when clinical manifestation is suggestive.

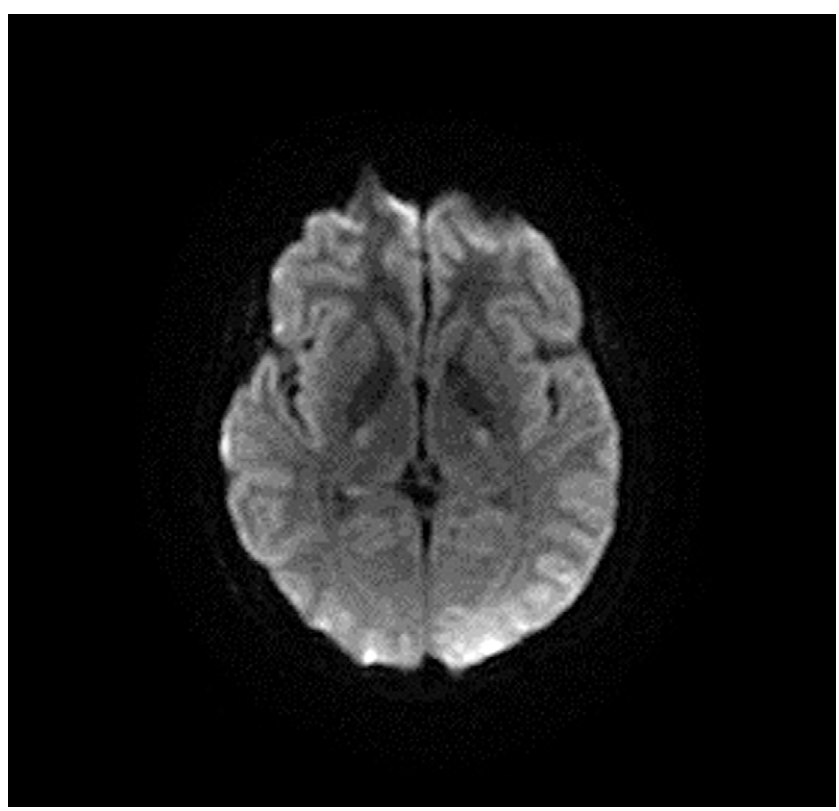

Figure 2. Diffusion-weighted imaging (DWI) typically shows lesser extensiveness of lesions in comparison to FLAIR sequence

\section{REFERENCES}

1.Hobson EV, Craven I, Blank SC. Posterior reversible encephalopathy syndrome: a truly treatable neurologic illness. Perit Dial Int. 2012; 32(6): 590-4. https://doi.org/10.3747/pdi.2012.00152

2. Fischer M, Schmutzhard E. Posterior reversible encephalopathy syndrome. J Neurol. 2017; 264(8): 1608-1616. https://dx.doi.org/10.1 007\%2Fs00415-016-8377-8

3. Li K, Yang Y, Guo D, et al. Clinical and MRI Features of Posterior Reversible Encephalopathy Syndrome With Atypical Regions: A Descriptive Study With a Large Sample Size. Front Neurol. 2020; 11: 194. https://dx.doi.org/10.3389\%2Ffneur.2020.00194

4. Parikh NS, Schweitzer AD, Young RJ, et al. Corticosteroid therapy and severity of vasogenic edema in posterior reversible encephalopathy syndrome. J Neurol Sci. 2017; 380: 11-15. https://doi.org/10.1016/j. jns.2017.06.044

5. Sudulagunta SR, Sodalagunta MB, Kumbhat M, et al. Posterior reversible encephalopathy syndrome(PRES). Oxf Med Case Reports. 2017; 2017(4): omx011. https://doi.org/10.1093/omcr/omx011

6. Kalaiselvan MS, Renuka MK, Arunkumar AS. Clinical Features and Outcomes of Patients with Posterior Reversible Encephalopathy Syndrome. Indian J Crit Care Med. 2017; 21(7): 453-456. https://doi. org/10.4103/ijccm.ijccm_79_17

7.Hinduja A. Posterior Reversible Encephalopathy Syndrome: Clinical Features and Outcome. Front Neurol. 2020; 11: 71. https://doi. org/10.3389/fneur.2020.00071

8. Lamy C, Oppenheim C, Mas JL. Posterior reversible encephalopathy syndrome. Handb Clin Neurol. 2014; 121: 1687-701. https://doi. org/10.1016/b978-0-7020-4088-7.00109-7

9. Okamoto K, Motohashi K, Fujiwara H, et al. PRES: Posterior Reversible Encephalopathy Syndrome. Brain Nerve. 2017; 69(2): 129-141. https:// doi.org/10.11477/mf.1416200653

10. Cordelli DM, Masetti R, Ricci E, et al. Life-threatening complications of posterior reversible encephalopathy syndrome in children. Eur J Paediatr Neurol. 2014; 18(5): 632-40. https://doi.org/10.1016/j.ejpn.2014.04.014 